\title{
Entinostat induces antitumor immune responses through immune editing of tumor neoantigens
}

\author{
Andrew S. Truong, ${ }^{1,2}$ Mi Zhou, ${ }^{1}$ Bhavani Krishnan, ${ }^{1}$ Takanobu Utsumi, ${ }^{1}$ Ujjawal Manocha, ${ }^{1}$ Kyle G. Stewart, ${ }^{1}$ Wolfgang Beck, ${ }^{1}$ \\ Tracy L. Rose, ${ }^{1,3}$ Matthew I. Milowsky, ${ }^{1,3}$ Xiaping He, ${ }^{1}$ Christof C. Smith, ${ }^{1}$ Lisa M. Bixby, ${ }^{1}$ Charles M. Perou, ${ }^{1,4,5}$ Sara E. Wobker, ${ }^{1,6}$ \\ Sean T. Bailey, ${ }^{1}$ Benjamin C. Vincent,, ${ }^{1,3,5,7}$ and William Y. Kim ${ }^{1,2,3,4}$ \\ 'Lineberger Comprehensive Cancer Center, ${ }^{2}$ Department of Pharmacology, ${ }^{3}$ Department of Medicine, ${ }^{4}$ Department of Cenetics, ${ }^{5}$ Computational Medicine Program, ${ }^{6}$ Department of Pathology, and \\ 7Department of Microbiology and Immunology, University of North Carolina at Chapel Hill (UNC), Chapel Hill, North Carolina, USA.
}

\begin{abstract}
Although immune-checkpoint inhibitors (ICIs) have been a remarkable advancement in bladder cancer treatment, the response rate to single-agent ICls remains suboptimal. There has been substantial interest in the use of epigenetic agents to enhance ICl efficacy, although precisely how these agents potentiate ICI response has not been fully elucidated. We identified entinostat, a selective HDAC1/3 inhibitor, as a potent antitumor agent in our immune-competent bladder cancer mouse models (BBN963 and BBN966). We demonstrate that entinostat selectively promoted immune editing of tumor neoantigens, effectively remodeling the tumor immune microenvironment, resulting in a robust antitumor response that was cell autonomous, dependent upon antigen presentation, and associated with increased numbers of neoantigen-specific T cells. Finally, combination treatment with anti-PD-1 and entinostat led to complete responses and conferred long-term immunologic memory. Our work defines a tumor cell-autonomous mechanism of action for entinostat and a strong preclinical rationale for the combined use of entinostat and PD-1 blockade in bladder cancer.
\end{abstract}

\section{Introduction}

In the United States, there will be an estimated 83,730 new cases of bladder cancer and an estimated 17,200 people will die of this disease in 2021 (1). Individuals with locally advanced and metastatic bladder tumors have an average 5-year survival rate of $15 \%$. After a decade-long drought of new therapeutics for advanced bladder cancer, the last few years have led to the approval of multiple antibodies targeting the PD-1/PD-L1 axis. Although immune-checkpoint inhibitors (ICIs), which are approved for patients that are refractory to, or ineligible for, cisplatin-based treatments, are a clear advance in bladder cancer treatment, only about $20 \%$ of these individuals respond to immunotherapy, emphasizing the continued need for drug development in this area (2-6).

Bladder cancer harbors a high frequency of somatic mutations (7). A fraction of these mutations can be expressed, processed, and presented as peptide neoantigens on the surface of tumor cells when bound by major histocompatibility complex molecules. These neoantigens can be targeted by T cells and thereby contribute to clearance of tumor cells by the adaptive-immune system. Tumor-mutational burden (TMB), as a correlate of predicted neoantigen burden, has generally been considered an important factor influencing the clinical benefit of ICIs $(5,8-11)$.

Authorship note: AST and MZ contributed equally to this work. Conflict of interest: The authors have declared that no conflict of interest exists. Copyright: ( 2021, American Society for Clinical Investigation. Submitted: March 30, 2020; Accepted: June 22, 2021; Published: August 16, 2021 Reference information: J Clin Invest. 2021;131(16):e138560. https://doi.org/10.1172/JCl138560.
Entinostat is a highly selective histone deacetylase 1 (HDAC1) and HDAC3 inhibitor. HDACs catalyze the removal of the acetyl groups on the lysine residues of histone tails. There are 18 HDAC enzymes in humans that are divided into 4 groups based on their homology to yeast HDACs (12). The removal of the acetyl groups from histone tails results in stronger binding between DNA and the core nucleosomal histones, limiting the access of transcriptional regulators to target genes. Therefore, unacetylated histones are typically associated with transcriptional inactivity. The expression of suppressed genes can be reinstated by inhibiting the activity of HDACs. Interestingly, many of the frequently mutated genes in bladder cancer are involved in modulating histone posttranslational modifications, including histone acetylation (i.e., EP300 and $C R E B B P$ ), as well as regulating nucleosome positioning (i.e., $A R I D 1 A)$, suggesting a potential contribution of epigenetic dysregulation in bladder cancer progression (13). Therefore, therapies that target epigenetic processes, such as HDAC inhibition, appear to be an attractive treatment for bladder cancer. Currently, there are multiple FDA-approved HDAC inhibitors for treating a variety of malignancies, such as cutaneous and peripheral T cell lymphoma and myeloma. Recently, the antitumor efficacy of HDAC inhibitors has been shown to go beyond stimulating apoptosis and arresting the cell cycle (14-17). For example, HDAC inhibitors have been shown to modulate the tumor immune microenvironment and enhance the response to PD-1 inhibition (18-23), but a limited body of work to date has explored the tumor cell-intrinsic effects of HDAC inhibition on antitumor immunity. Specifically, previously published work suggests that entinostat can decrease the populations of immune-suppressive cells such as myeloid-derived suppressive cells (MDSCs) and $\mathrm{FOXP}^{+}$Tregs in murine models 
A

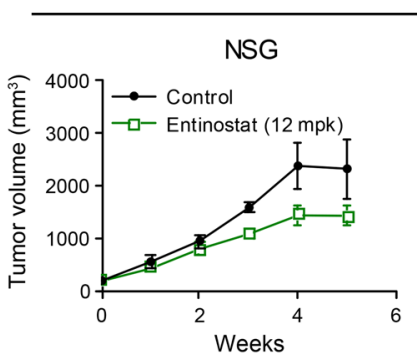

BBN963
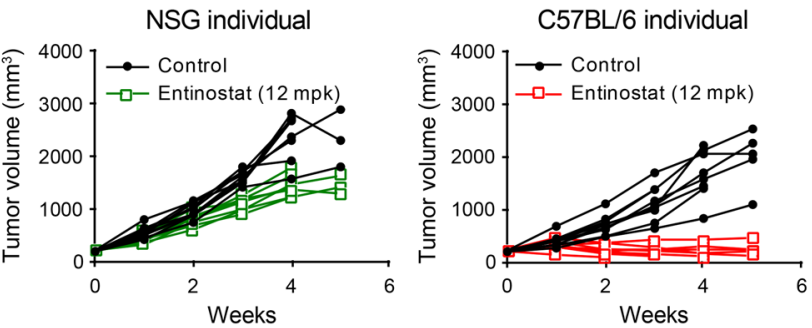

B

BBN966
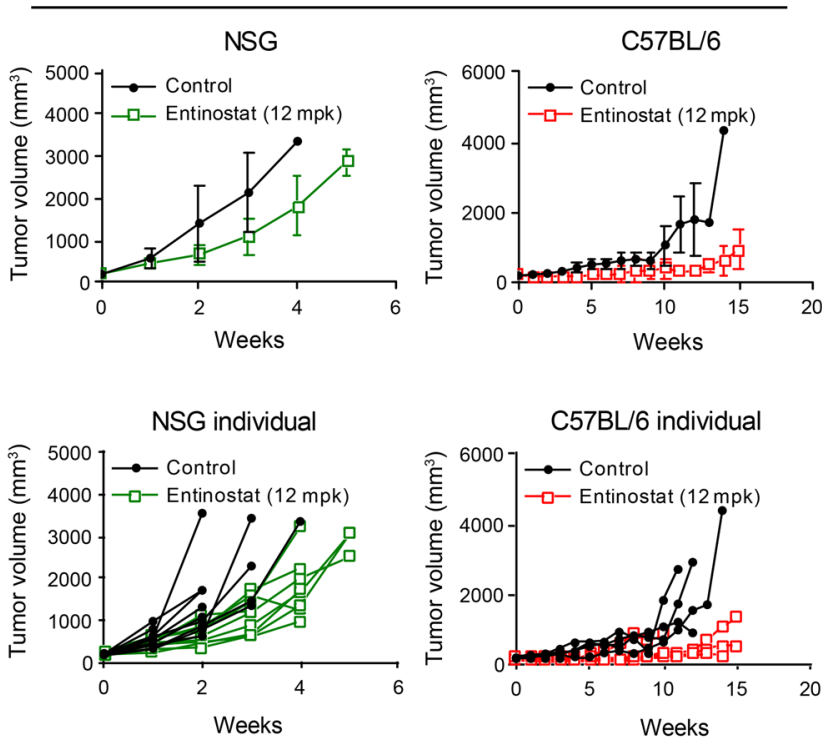

of lung, colorectal, mammary, and renal cell carcinoma $(19,20)$. Additionally, the inhibitor can enhance the antitumor activity of NK cells by upregulating the NKG2D receptor and its canonical ligands on NK cells and tumor cells, respectively (23).

Here, we evaluated the antitumor efficacy and cell-autonomous mechanism of action of the selective HDAC1 and HDAC3 inhibitor entinostat. In our previously reported immune-competent murine models of high-grade muscle invasive bladder cancer (24), we found that entinostat exhibited robust in vivo antitumor activity in immune-competent but not immune-compromised hosts. RNA-Seq analysis of entinostat-treated tumors demonstrated increased immune gene signature expression with flow cytometry, confirming an increase in $\mathrm{CD}^{+}$effector memory T cells. More importantly, our study unveiled what we believe is the first tumor cell-intrinsic mechanism of action of entinostat that includes remodeling of the expressed tumor neoantigen landscape. Entinostat treatment of BBN963 tumors in C57BL/6 hosts
Figure 1. Entinostat promotes a robust antitumor response in immune-competent C57BL/6 mice. (A) Average BBN963 tumor volume in response to entinostat in immune-deficient NSC mice and immune-competent C57BL/6 mice with tumor volume of each individual mouse shown in separate graphs. $n=9-10$ mice. (B) Average BBN966 tumor volume in response to entinostat in immune-deficient NSG mice and immune-competent C57BL/6 mice with tumor volume of each individual mouse shown in separate graphs. $n=5-6$ mice. Data are represented as mean $\pm S D$.

caused a striking decrease in the expression of predicted tumor neoantigens that was not seen in entinostat-treated tumors grown in NOD/ SCID/IL2r $\gamma^{\text {null }}$ (NSG) mice, consistent with selective immune editing in immunocompetent models. Combination treatment of entinostat and anti-PD-1 effectively eliminated BBN tumors in mice and promoted long-term immunologic memory. In aggregate, these data support the hypothesis that entinostat mediates antitumor effects and long-term immunologic memory through enhanced neoantigen expression and antigen-driven adaptive-immune responses.

\section{Results}

Entinostat has antitumor efficacy in immune-competent murine models of bladder cancer. To evaluate the in vivo efficacy of entinostat in an immune-competent setting, we implanted BBN963 and BBN966 cells subcutaneously into the flanks of immune-deficient NSG mice or immune-competent C57BL/6 mice. Upon tumor formation (200 $\mathrm{mm}^{3}$ in volume), mice were randomized to treatment with either vehicle or entinostat (12 $\mathrm{mg} / \mathrm{kg}$ in chow). In the NSG mice, entinostat inhibited 30\% of BBN963 tumor growth $(P<$ $0.001, t$ test; Figure $1 \mathrm{~A})$ after 5 weeks of treatment. However, in the C57BL/6 mice, the effect of entinostat was more robust $(90 \%, P<$ $0.0001, t$ test; Figure 1A). A similar pattern of antitumor response was observed in BBN966 cell line-derived tumors (Figure 1B). The selective response to entinostat treatment in immunocompetent C57BL/6 mice suggested that the immune system played a key role in triggering the observed antitumor effect of entinostat.

Entinostat promotes an inflamed tumor microenvironment. To begin to evaluate the mechanism underlying robust in vivo antitumor efficacy of entinostat in immunocompetent mice, we assessed changes in gene expression of tumors grown in C57BL/6 mice with and without exposure to entinostat. Specifically, we generated mice bearing subcutaneous BBN963 tumors. Once tumors reached $200 \mathrm{~mm}^{3}$ in volume, mice were randomized to 3 groups: (a) baseline (tumors harvested immediately), (b) vehicle, and (c) entinostat treatment. When entinostat-treated tumors reached approximately $100 \mathrm{~mm}^{3}$, both vehicle- and entinostat-treated tumors were harvested and RNA extracted, and transcriptome profiling was performed by RNA-Seq (Figure 2A). Treatment with entinostat induced robust changes in gene expression $(23000-$ 4000 genes) when compared with baseline or vehicle-treated tumors (Figure 2B). There was minimal change in gene expression when comparing the baseline and vehicle-treated tumors, indicating that the difference in tumor size alone did not drive significant gene expression changes in this setting. Next, we used Ingenuity Pathway Analysis (IPA) to contextualize the RNA-Seq data comparing the vehicle-treated and entinostat-treated tumors. We found that the majority of the pathways upregulated were processes involved in immune response (Figure 2C). 
A

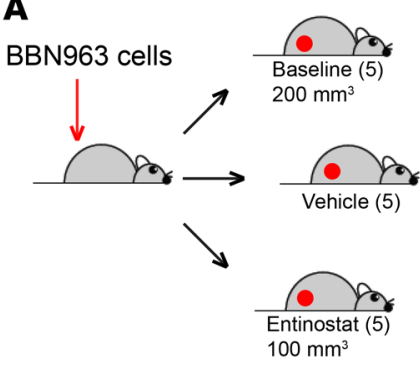

C

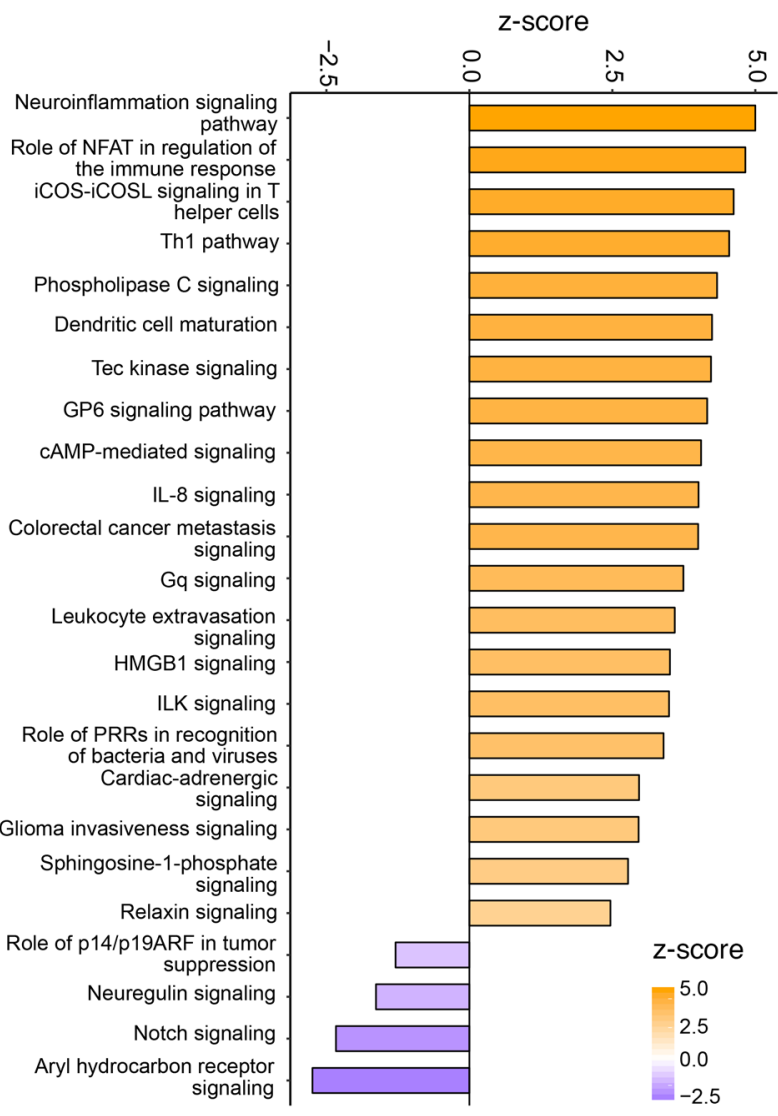

E

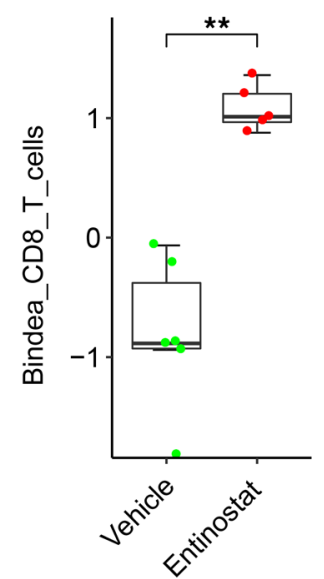

B

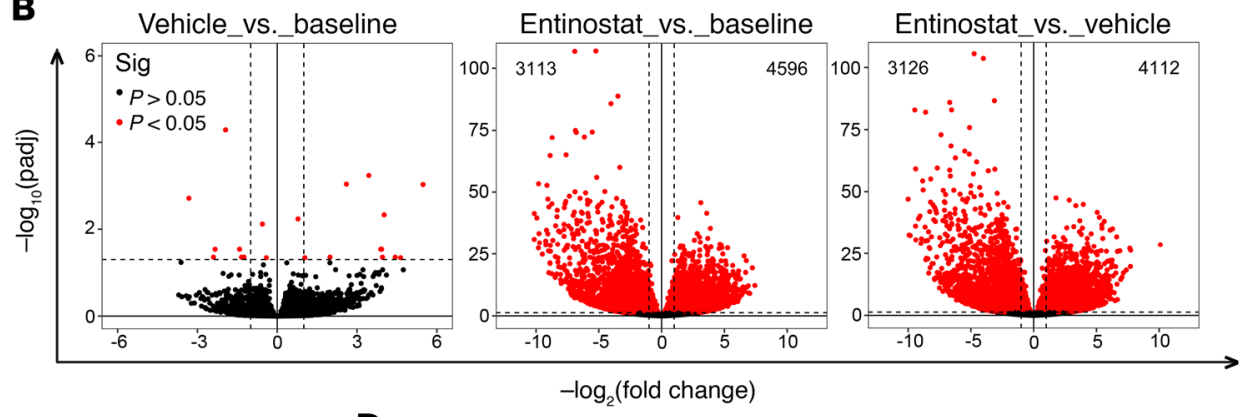

D

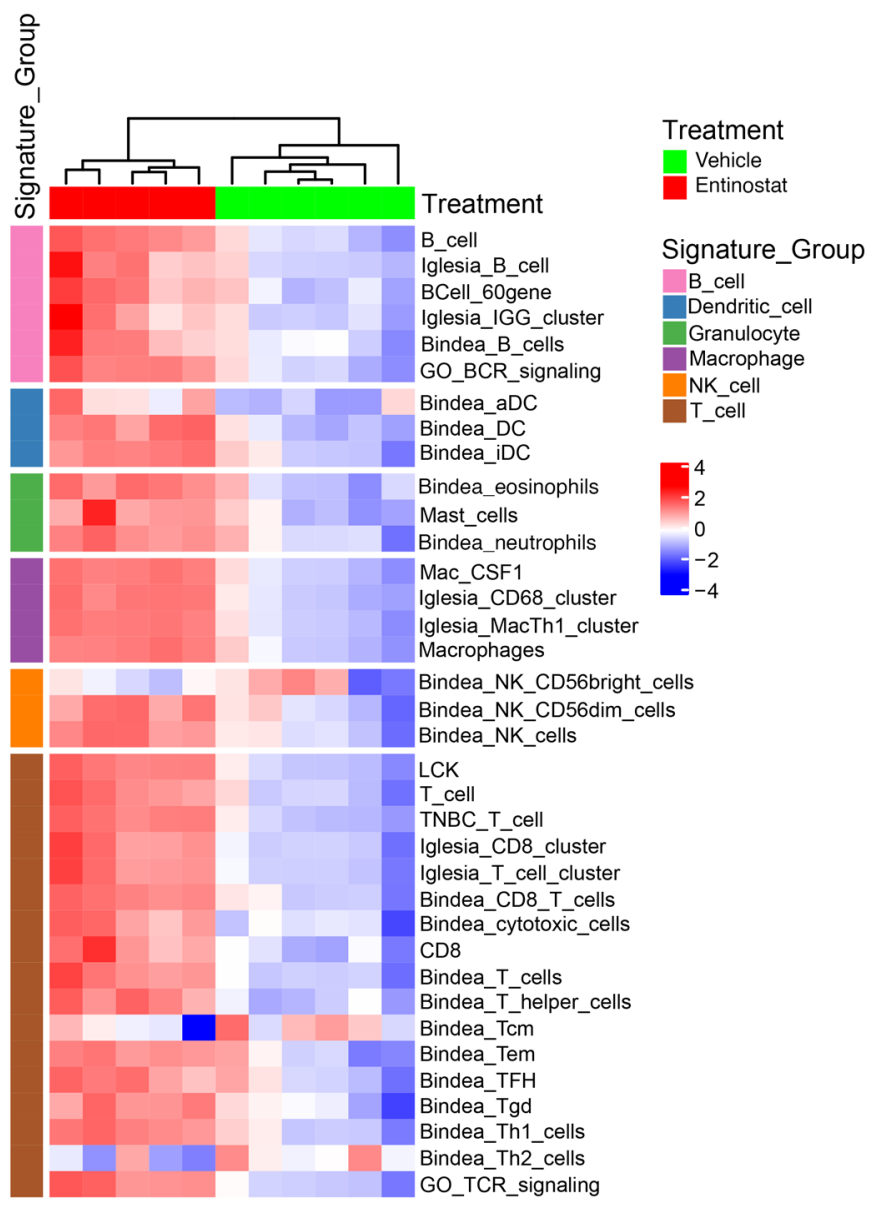

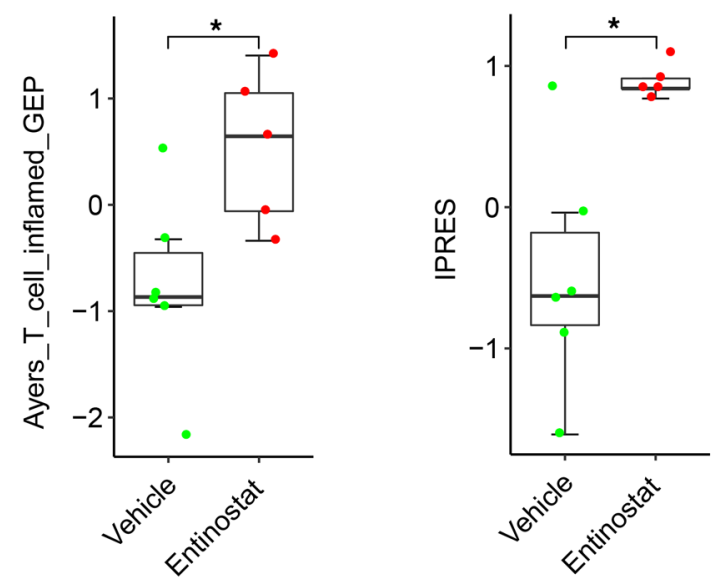


Figure 2. Entinostat promotes an inflamed tumor microenvironment. (A) Schematic showing how BBN963 tumors were collected for RNA-Seq. (B) Volcano plots of $\log _{2}$ fold change of median gene expression and $-\log _{10} P$ value of gene expression between the indicated treatment groups. Dashed line across plots corresponds to a significance threshold of $P=0.05$. Significance was calculated using $t$ test. (C) IPA plot of activated pathways in entinostat-treated tumors relative to vehicle-treated tumors. (D) Heatmaps of unsupervised clustering of vehicle- $(n=6)$ and entinostat-treated $(n=5)$ tumors across previously established immune gene signatures (ICS). (E) Box plots of indicated immune gene signatures. Significance was calculated using Mann-Whitney $U$ test. Data are represented as mean $\pm \mathrm{SD} .{ }^{*} P<0.05 ;{ }^{* *} P<0.01$.

Immune gene expression signatures derived from previously published studies were compared between the vehicle- and entinostat-treated tumors. By analyzing the RNA-Seq data, we found that treatment of BBN963 tumors with entinostat resulted in a broad increase in expression of immune gene signatures in comparison with what occurred in vehicle-treated tumors (Figure 2D) that was significant upon direct testing (examples shown in Figure $2 \mathrm{E}$ ). Taken together, these results strengthened our hypothesis that entinostat modulates the tumor immune microenvironment, promoting a T cell-inflamed phenotype.

Entinostat increases antigen-experienced $T$ cell responses and decreases immune-suppressive populations. To validate the increase in expression of immune gene signatures in entinostat-treated tumors, we performed flow cytometry to quantify the relative frequency of different immune cell populations. Entinostat treatment of BBN963 tumors began when they reached approximately 500 $\mathrm{mm}^{3}$ in volume. Tumors were harvested after 7 days of treatment. While we did not see significant changes in $\mathrm{CD} 8^{+} \mathrm{T}$ cell numbers, we noted an increase of CD8 memory cells, the majority of which were effector memory $\mathrm{T}$ cells (Figure 3A). We did not see significant changes in $\mathrm{CD} 4^{+}$memory populations. Additionally, in congruence with prior work, we observed that entinostat treatment decreased suppressive cell types, such as MDSCs and Tregs (refs. 19, 20, and Supplemental Figure 1; supplemental material available online with this article; https://doi.org/10.1172/JCI138560DS1). Supplemental Figure 1 includes flow cytometry results of the entire panel of immune cells analyzed. Given the presence of both granulocytic and monocytic MDSCs (M-MDSCs) in bladder tumors (Supplemental Figure 2A), we evaluated these independently for their capacity to suppress $\mathrm{T}$ cell expansion when stimulated with anti-CD3 and anti-CD28 antibodies to mimic T cell receptordependent $\mathrm{T}$ cell activation. We found that M-MDSCs, but not granulocytic MDSCs (G-MDSC), were potently suppressive of T cell expansion (Figure 3, B and C). M-MDSCs were also selectively depleted by entinostat (Figure 3, D and E, and Supplemental Figure 2B). These results show that entinostat modulated the tumor immune microenvironment, allowing for the expansion of an antigen-experienced $\mathrm{T}$ cell response as well as decreasing immune-suppressive populations, such as Tregs and M-MDSCs.

Entinostat promotes selective immune editing based on tumor neoantigen expression. Prior work has shown that entinostat can promote acetylation of STAT3 to regulate its transcriptional activity (25). We, however, did not see changes in STAT3 acetylation in our BBN963 cells treated with entinostat in vitro (Supplemental Figure 3 ). Given the known role of histone acetylation on tran- scription, we hypothesized that entinostat might alter cell-autonomous transcriptional changes to promote antitumor immunity through the RNA expression levels of tumor-associated antigens. We examined changes in RNA expression of neoantigens, cancer testis antigens (CTAs), and murine endogenous retroviruses (mERVs) in control and entinostat-treated tumors with the notion that immune editing should decrease the expression of tumor antigens in immune-competent, but not immune-deficient, mice. We did not see any consistent pattern of effects of entinostat on CTA or mERV expression in entinostat-treated tumors grown in B6 mice (Figure 4A), suggesting that differential expression of CTAs or mERVs was not mediating the antitumor effects of entinostat.

We had previously predicted tumor neoantigens in BBN963 cells (24). From whole-exome sequencing (WES), our neoantigen prediction pipeline identified 3902 potential neoantigens in BBN963 cells (Supplemental Figure 4). We used the RNASeq data from our baseline and vehicle- and entinostat-treated tumors to assess how expression of these predicted neoantigens changed with entinostat treatment. Examination of baseline tumors showed that they expressed 922 of the 3902 predicted neoantigens at the RNA level. We saw that the majority of the 922 expressed neoantigens had persistent RNA expression in vehicle-treated tumors. This important control suggests that neither merely growing BBN963 cells in vivo nor tumor size (baseline $=198 \mathrm{~mm}^{3}$ versus vehicle $=2012 \mathrm{~mm}^{3}$ ) dramatically affects predicted neoantigen expression. In contrast, the majority of predicted class I MHC-expressed neoantigens $(n=745)$ lost RNA expression in entinostat-treated tumors (Supplemental Figure 4, A and B), consistent with the notion that entinostat (through upregulated expression of neoantigens) induced an antigen-driven immune response to promote "immune editing" $(26,27)$ in the BBN963 tumors grown in immune-competent C57BL/6 hosts. We found a similar pattern of changes in predicted neoantigen expression of peptides presented in the context of class II MHC (Supplemental Figure 4C) in C57BL/6 mice, but not in NSG mice (Supplemental Figure 4D).

We confirmed by quantitative reverse-transcriptase PCR (qRT-PCR) that a representative set of predicted neoantigens was significantly decreased in entinostat-treated tumors (Figure 4B) and that their expression increased in a dose-dependent manner in BBN963 cells treated in vitro with entinostat (Figure 4C). Further supporting the notion that entinostat promotes immune editing, a parallel analysis of expressed neoantigens in entinostat-treated tumors grown in NSG mice showed no expression changes of predicted neoantigens (Figure 4A and Supplemental Figure 4, B and D). Moreover, consistent with immune editing, we saw that the total number of predicted neoantigens was significantly lower at the DNA level in entinostat-treated tumors shown in Figure 1, C and $\mathrm{D}$, and Figure 4D. In aggregate, these findings highly suggest that entinostat mediates an antigen-driven immune response to predicted neoantigens.

An antigen-driven immune response would be predicted to correlate with increased TCR sharing across replicate tumors. To see whether this was true in our model, we performed TCR repertoire profiling of tumor-infiltrating $\mathrm{T}$ cells in entinostat-treated BBN963 tumors. We observed a significantly increased $\mathrm{T}$ cell receptor $\alpha$ (TRA) clonotype sharing in entinostat-treated tumors 
A

CD45+

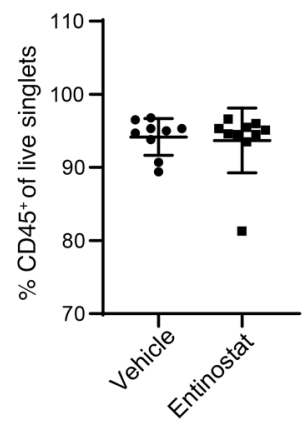

CD8 total memory
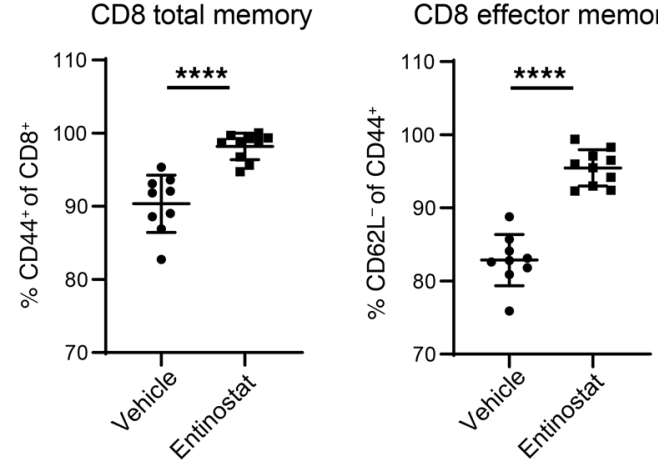

B
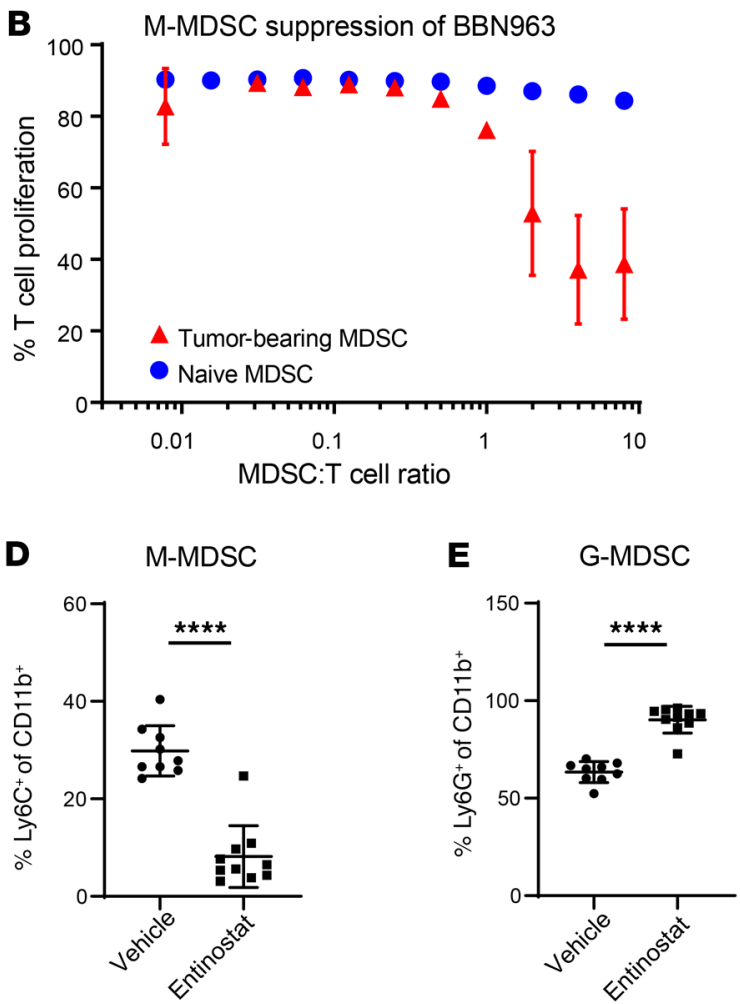

relative to vehicle-treated controls (Figure 4, E and F), consistent with the notion that entinostat mediates an antigen-driven, $\mathrm{T}$ cellmediated immune response. Moreover, we determined whether entinostat treatment increased tetramer staining of $\mathrm{CD}^{+}$splenocytes of BBN963 tumor-bearing mice. Specifically, we generated neoantigen-specific tetramers to label $\mathrm{CD}^{+} \mathrm{T}$ cells from spleno-
CD8 T cells

CD4 T cells
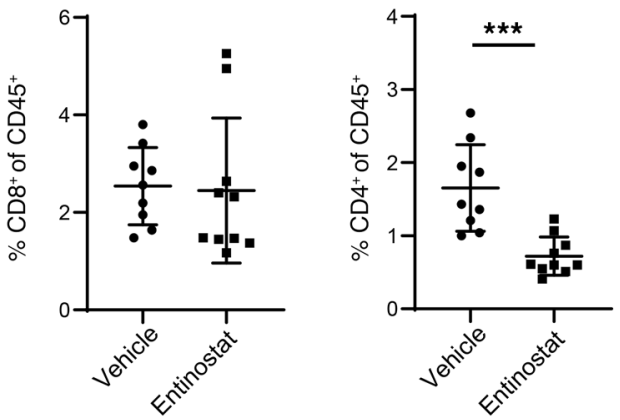

CD4 effector memory

Treg

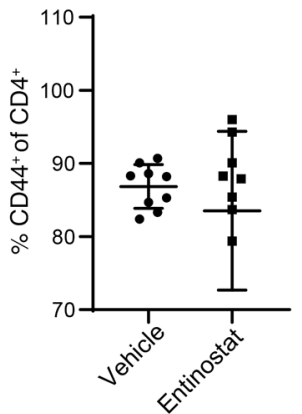

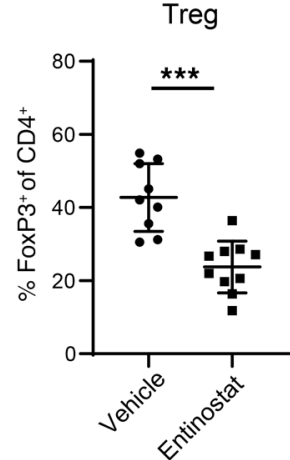

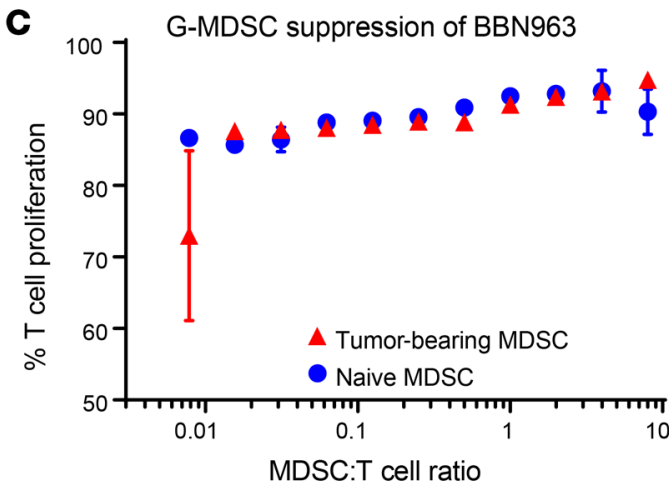

Figure 3. Entinostat increases antigen-experienced $\mathrm{T}$ cell responses and decreases immune-suppressive populations. (A) Percentages of different representative immune cells in vehicle-treated $(n=9)$ and entinostat-treated $(n=10)$ tumors using flow cytometry. (B) T cell proliferation when cultured with increasing numbers of M-MDSCs per T cell. (C) T cell proliferation when cultured with increasing numbers of G-MDSCs per T cell. (D) Proportion of lineage-negative CD11 b+ cells that are Ly6C- ${ }^{-}$and Ly6 $\mathrm{C}^{+}$ (M-MDSC) in vehicle-treated $(n=9)$ and entinostat-treated $(n=10)$ tumors. (E) Proportion of lineage-negative CD11b+ cells that are Ly6C+ (G-MDSC) in vehicle-treated $(n=9)$ and entinostat-treated $(n=10)$ tumors. Significance was calculated using Mann-Whitney $U$ test. Data are represented as mean \pm SD. ${ }^{* *} P<0.001 ;{ }^{* * *} P<0.0001$.

cytes of mice bearing BBN963 tumors treated with either vehicle or entinostat. Splenocytes were isolated from the mice and stained with the tetramers as well as antibodies against $\mathrm{CD} 3$ and $\mathrm{CD} 8$. All 3 neoantigens (Car12, Grin1, and Has2) showed higher levels of tetramer-positive $\mathrm{CD} 3^{+} \mathrm{CD} 8^{+} \mathrm{T}$ cells in entinostat-treated mice, with increases in Car12 and Grin1 tetramers being significant. We 
A
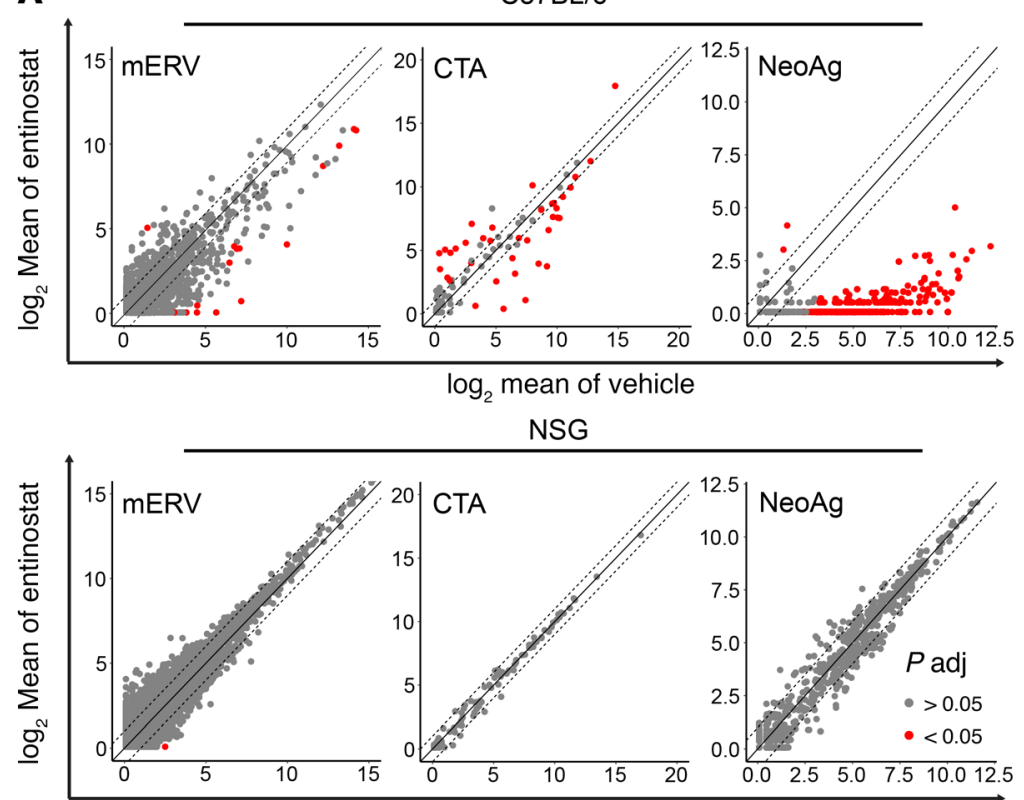

$\log _{2}$ mean of vehicle
B

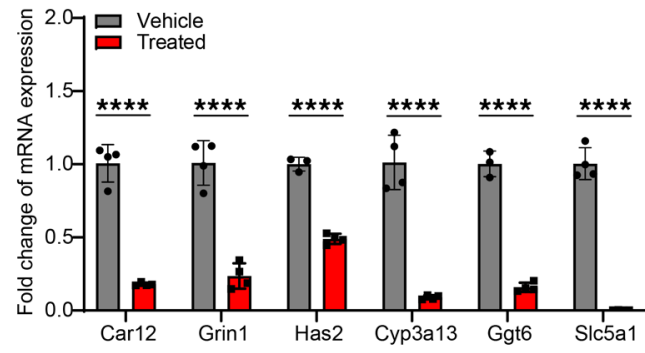

C

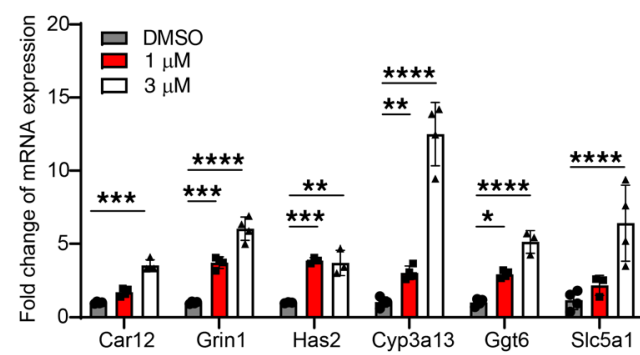

G

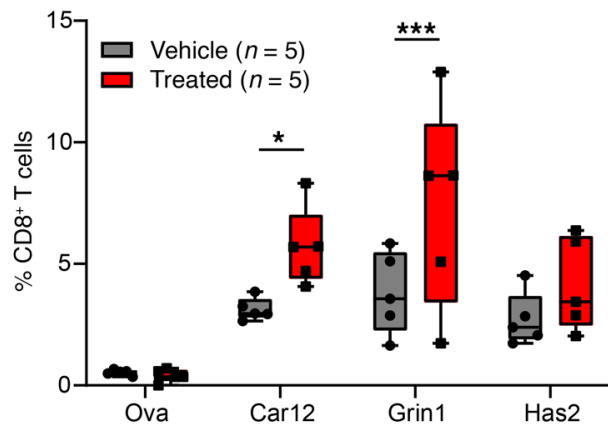

Figure 4. Entinostat promotes a neoantigen-driven antitumor response. (A) Scatter plots of $\log _{2}$ mean of mERV, CTA, and neoantigen (NeoAg) expression in entinostat-treated versus vehicle-treated tumors in immune-competent C57BL/6 mice (upper panel) and in immune-deficient NSG mice (lower panel). (B) mRNA expression of selective neoantigens in BBN963 tumors in C57BL/6 mice treated with vehicle $(n=4)$ or entinostat $(n=4)$. Significance was calculated by $t$ test. (C) mRNA expression of selective neoantigens in BBN963 cells treated with DMSO or entinostat in vitro $(n=4)$ for 72 hours. Significance was calculated using 2-way ANOVA followed by Dunnett's multiple-comparison test. (D) Total neoantigen count derived from WES of DNA isolated from BBN963 tumors in C57BL/6 mice treated with vehicle $(n=3)$ or entinostat $(n=3)$. Significance was calculated by Mann-Whitney $U$ test. $(\mathbf{E})$ Heatmap and $(\mathbf{F})$ quantification of T cell receptor clonotype sharing between vehicle- and entinostat-treated tumors, derived from whole-tumor RNA-based T cell receptor sequencing. Significance was calculated by Mann-Whitney $U$ test. (G) Percentage of CD8 ${ }^{+} T$ cells that were labeled positive with indicated tetramers in BBN963 tumors in C57BL/6 mice treated with vehicle $(n=5)$ or entinostat $(n=5)$. Significance was calculated using $t$ test. Data are represented as mean \pm SD. Box plots represent mean \pm minimum and maximum values. ${ }^{*} P<0.05 ;{ }^{* *} P<0.01 ;{ }^{* *} P<0.001 ;{ }^{* * * *} P<0.0001$.

did not see any significant tetramer staining against OVA in vehicle- or entinostat-treated mice, demonstrating the effect was specific to predicted neoantigens (Figure 4G).

$B 2 m$ CRISPR partially rescues the antitumor effect of entinostat. To determine whether neoantigen presentation by MHC molecules is necessary for the antitumor effect of entinostat, we depleted MHC class I in BBN963 cells (BBN963-sgB2m) by knocking out $\beta_{2}$ microglobulin (B2m) using CRISPR/Cas9. We tested 9 different guide RNAs (gRNAs) and found that the gRNAs no. 4 and no. 5 were able to successfully knock out B2m as confirmed by Western blotting, and the depletion of cell surface MHC class I was con- firmed by FACS (Figure 5, A and B). Polyclonal BBN963-sgB2m (no. 4) and BBN963-EV (empty CRISPR/Cas9 construct without gRNA) cells were implanted into C57BL/6 mice subcutaneously. Upon tumor formation $\left(200 \mathrm{~mm}^{3}\right)$, mice were randomized to vehicle or entinostat treatment. As previously observed, entinostat robustly inhibited the progression of BBN963-EV tumors. In contrast, the depletion of MHC class I antigen presentation in the BBN963-sgB2m tumors significantly abrogated the antitumor effect of entinostat in comparison with that in entinostat-treated BBN963-EV tumors (Figure 5, C and D). Parallel experiments were conducted using BBN966 cells with B2m CRISPR (Supple- 
A

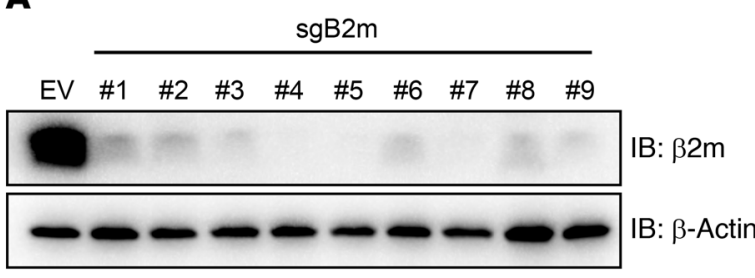

C

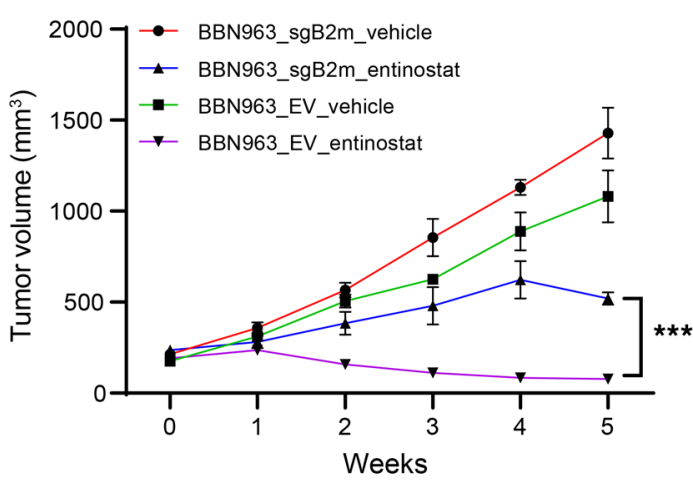

$\mathbf{E}$

BBN966

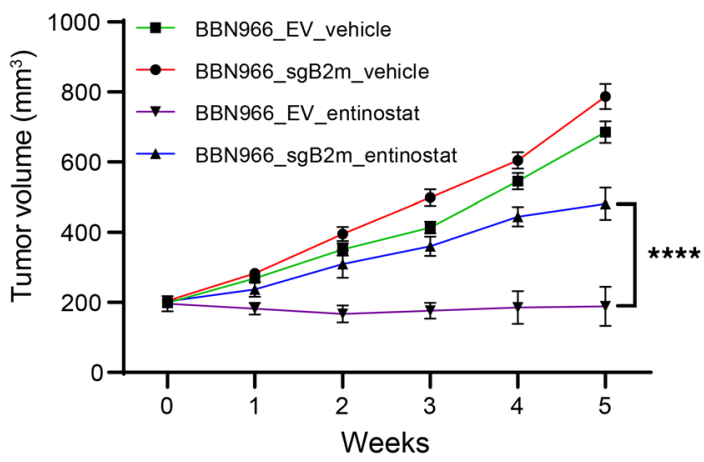

G

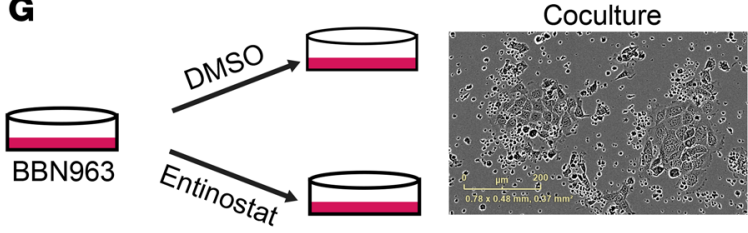

H

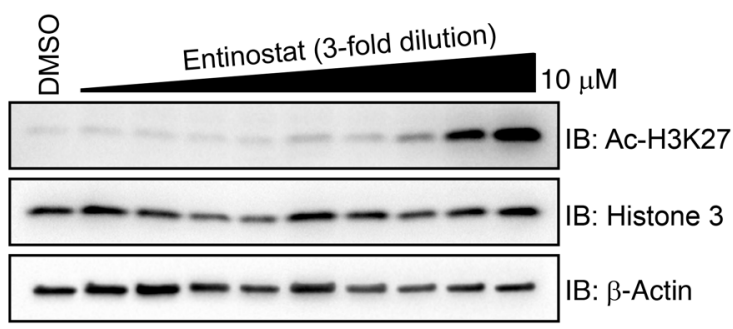

B

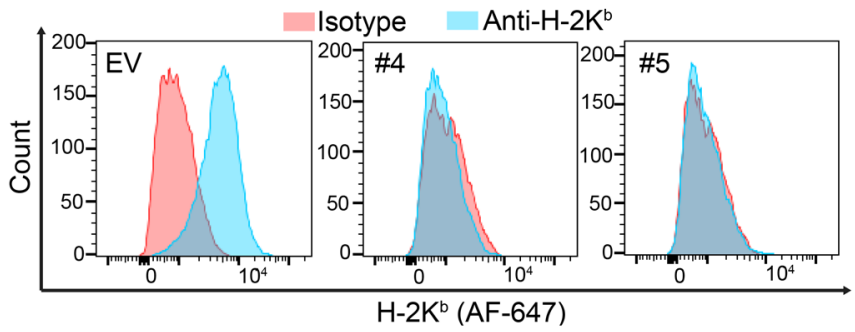

D

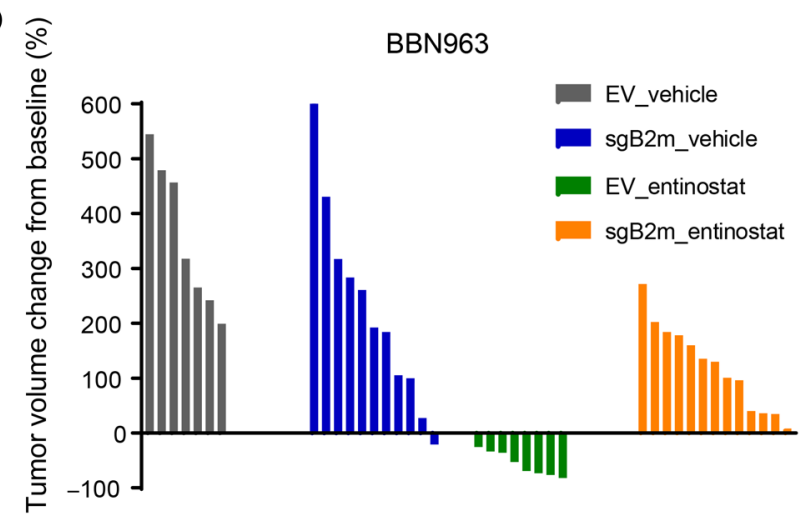

$\mathbf{F}$

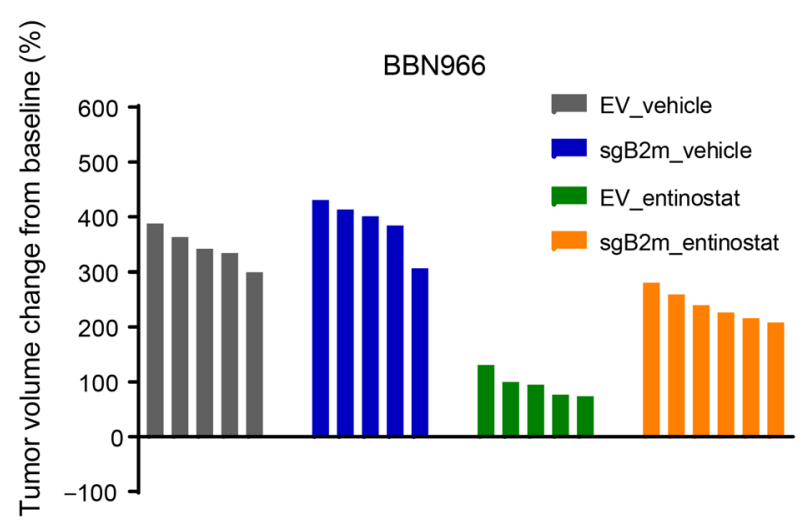

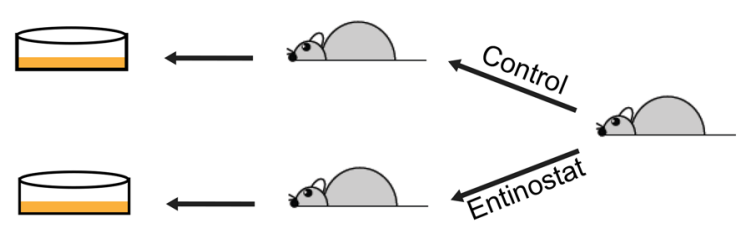

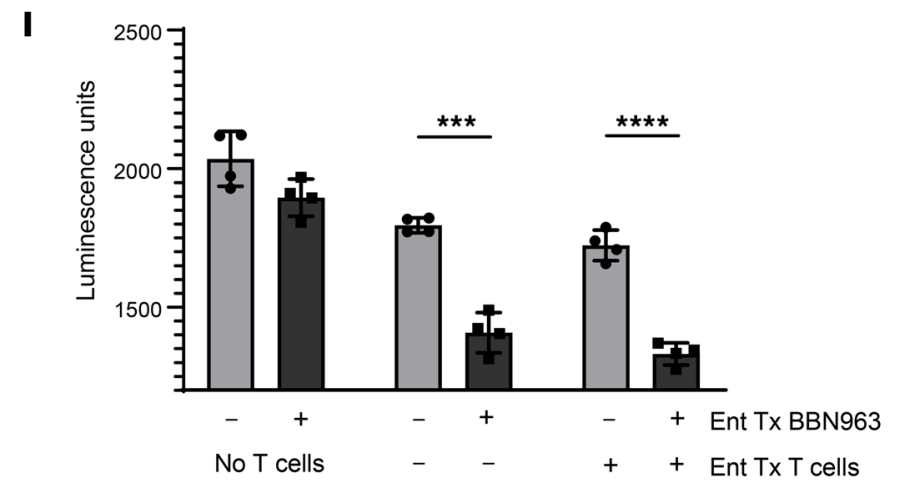


Figure 5. B2m CRISPR partially rescues the antitumor effect of entinostat. (A) Immunoblot of BBN963 cells with B2m knockout using 9 different individual sgRNA constructs. (B) Flow cytometry graph of $\mathrm{H}-2 \mathrm{~K}^{\mathrm{b}}$ (class $1 \mathrm{MHC}$ ) cell-surface expression in B2m-knockout BBN963 cells in A with sgRNA no. 4 and no. 5. (C) Average volume of BBN963_EV (empty vector) and BBN963_ sgB2m (B2m knockout) tumors in response to entinostat (12 mpk) in C57BL/6 mice. $n=7-13$ mice per group. Significance was calculated using 2-way ANOVA followed by Tukey's multiple-comparison test. (D) Waterfall plot of individual tumor volumes in $\mathbf{C}$ at end points relative to pretreatment baseline. End points were tumor burden and ulceration. (E) Average volume of BBN966_EV (empty vector) and BBN966_sgB2m (B2m knockout) tumors in response to entinostat (12 mpk) in C57BL/6 mice. $n=5-6$ mice per group. Significance was calculated using 2-way ANOVA followed by Tukey's multiple-comparison test. (F) Waterfall plot of individual tumor volumes in $\mathbf{E}$ at end points relative to pretreatment baseline. End points were tumor burden and ulceration (G) Schematic of how the in vitro T cell killing assays were set up. (H) Immunoblots of BBN963 cells treated with various concentrations of entinostat for 24 hours and blotted for the indicated antibodies. (I) Bar graph showing the result of $\mathrm{T}$ cell killing assays. CellTiter-Glo was used to quantify viable BBN963 cells at the end of the 72-hour coculture. Significance was calculated using $t$ test. Data are represented as mean $\pm \mathrm{SD} .{ }^{* * *} P<0.001 ;{ }^{* * *} P<0.0001$.

mental Figure 5A) or control cells implanted into B6 mice. Similarly to BBN963 cells, the antitumor effect of entinostat was partially abrogated by B2m CRISPR (Figure 5, E and F). These results confirm that antigen presentation on the surface of BBN963 and BBN966 cells is necessary for entinostat efficacy.

Entinostat treatment of tumor cells promotes ex vivo $T$ cell killing. While our data implicating entinostat in mediating increased tumor neoantigen-targeted immunity suggest a cell-autonomous mechanism of entinostat efficacy, we noted that entinostat has been previously described as directly affecting $\mathrm{T}$ cell function. To further substantiate our claims as well as to assess whether entinostat promotes $\mathrm{T}$ cell killing in this model, we performed a coculture assay by mixing entinostat- or vehicle-treated BBN963 cells with T cells from BBN963 tumor-bearing mice treated with entinostat or vehicle (Figure $5 G$ ). Specifically, we first determined the optimal dose for in vitro entinostat treatment by treating BBN963 cells with a range of entinostat doses to determine the lowest dose of entinostat $(1 \mu \mathrm{M})$ that inhibited global H3K27Ac of BBN963 cells $(1 \mu \mathrm{M}$ : Figure $5 \mathrm{H})$. We then cocultured these cells with $\mathrm{CD}^{+} \mathrm{T}$ cells isolated from the spleens of BBN963 tumor-bearing mice treated with entinostat or vehicle for 4 weeks. After 72 hours, T cells were washed off and CellTiter-Glo was used to quantify the presence of viable BBN963 cells. We observed a minimal, but not statistically significant, difference $(P>0.05)$ in BBN963 cell viability in the absence of T cells, suggesting that entinostat has minimal cell-autonomous antitumor efficacy (Figure 5I). In contrast, the viability of entinostat-pretreated BBN963 cells was significantly reduced in comparison with DMSO-pretreated BBN963 cells when cocultured with $\mathrm{CD}^{+} \mathrm{T}$ cells from BBN963 tumor-bearing mice $(P<$ 0.001). Importantly, we did not see any significantly increased BBN963 killing when the source of $\mathrm{CD}^{+} \mathrm{T}$ cells was from entinostat-treated, BBN963 tumor-bearing mice. Furthermore, we noted that $\mathrm{CD}^{+} \mathrm{T}$ cells from WT (non-tumor-bearing) mice could also effectively kill entinostat-treated BBN963 cells (Supplemental Figure $5 \mathrm{~B})$. The level of cleaved caspase- 3 was not increased by $1 \mu \mathrm{M}$ entinostat treatment in BBN963 cells in vitro, suggesting that entinostat at this concentration does not trigger apoptosis to enhance
T cell-mediated tumor cell killing (Supplemental Figure 5C). These results in aggregate demonstrate that entinostat pretreatment of BBN963 cells, but not $\mathrm{T}$ cells, promotes $\mathrm{CD}^{+} \mathrm{T}$ cell killing and are consistent with the notion that entinostat provokes antitumor immunity in a cell-autonomous manner.

Entinostat plus PD-1 inhibition promotes effective long-term antitumor immunity. Correlative studies from clinical trials have consistently shown a number of immunogenomic predictors of response to PD-1 or PD-L1 blockade, including higher levels of PD-L1 as well as high levels of a number of immune gene signatures, such as $\mathrm{CD}^{+} \mathrm{T}$ cell signatures and IFNG (28). We have already shown that entinostat-treated BBN963 tumors have higher levels of $\mathrm{CD}^{+} \mathrm{T}$ cell signature (Figure 2B). Examination of CD274 (PD-L1) transcript expression (Figure 6A) demonstrated that PDL1 expression was elevated in entinostat-treated tumors as well and appeared to be upregulated primarily in tumor cells (Supplemental Figure 6). The immunogenomic changes we saw from entinostat may therefore predict for enhanced efficacy of immune-checkpoint therapy.

We tested this hypothesis directly in BBN963 cell line-derived tumors grown subcutaneously in C57BL/6 mice. Once tumors reached $200 \mathrm{~mm}^{3}$, they were randomized into treatment groups with vehicle plus control IgG, entinostat, anti-PD-1 (clone RPM114), and entinostat plus anti-PD-1. The combination of entinostat plus anti-PD1 significantly outperformed each agent alone (Figure $6, \mathrm{~B}$ and C). Indeed, we observed 6 of 9 cases of complete response (CR) in the combination treatment, while we observed only 1 of 9 cases of CR in the group treated with entinostat only (Figure 6, $\mathrm{C}$ and $\mathrm{D})$. To our surprise, we did not see significant shrinkage in tumor volume in the anti-PD1 group, as we have previously reported (24). However, necropsy analysis indicated that, while anti-PD1-treated tumors continued to enlarge, all of these tumors had undergone central necrosis (Supplemental Figure 7). To evaluate the durability of combination treatment, we discontinued treatment of mice after the tumors had obtained a CR and then monitored the animals for any tumor regrowth. We observed no tumor formation over 8 weeks in any of the animals that had CR. To determine whether the entinostat plus anti-PD-1 treatment conferred long-term immunologic memory, we rechallenged the animals with BBN963 cell injections. In age-matched control mice that had never been implanted with BBN963 cells or received drug treatments, we observed a take rate of $80 \%$. However, in the mice previously treated with entinostat plus anti-PD-1, the BBN963 rechallenge did not result in any tumors (Figure 6E). These results indicate that entinostat plus anti-PD-1 combination therapy is able to eliminate BBN963 cells completely and promote long-term immunologic memory.

\section{Discussion}

Considerable progress in bladder cancer treatment has been made in recent years with the approval of ICIs and the antibody drug conjugate enfortumab vedotin as well as targeted therapy for FGF receptor-altered (FGFR-altered) tumors. Nonetheless, the majority of patients still have a suboptimal response to these therapies, highlighting the need for continued development of more effective drugs and, in particular, drugs that synergize with immune-checkpoint inhibition. In this study, we demonstrate the 
A

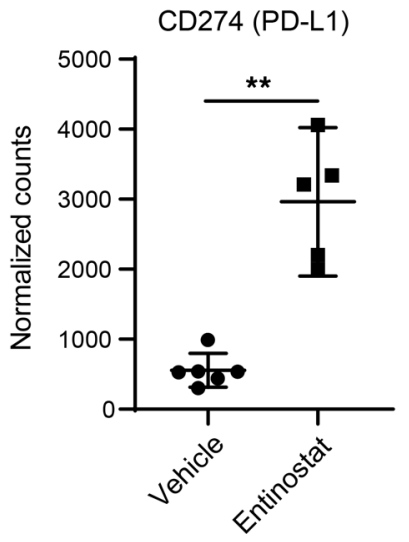

C

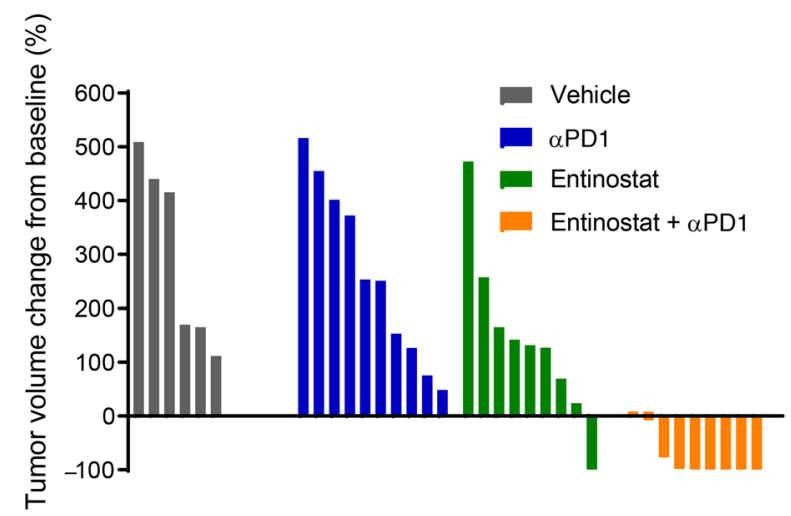

B

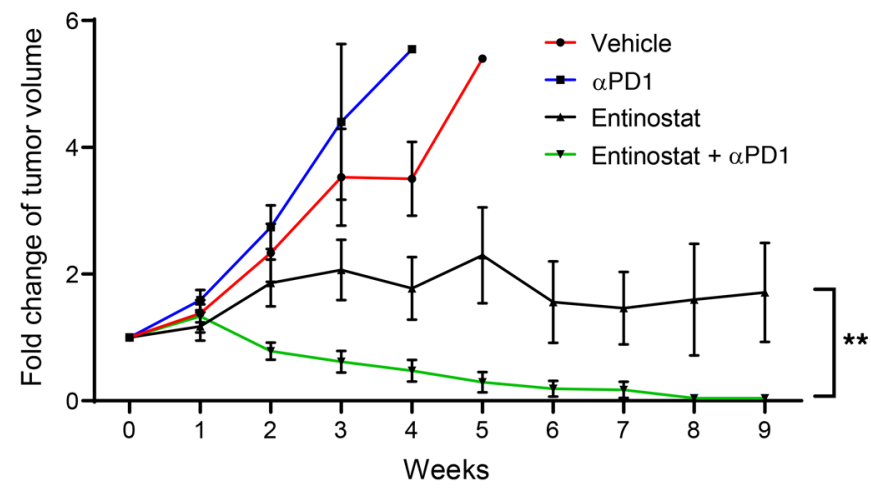

D

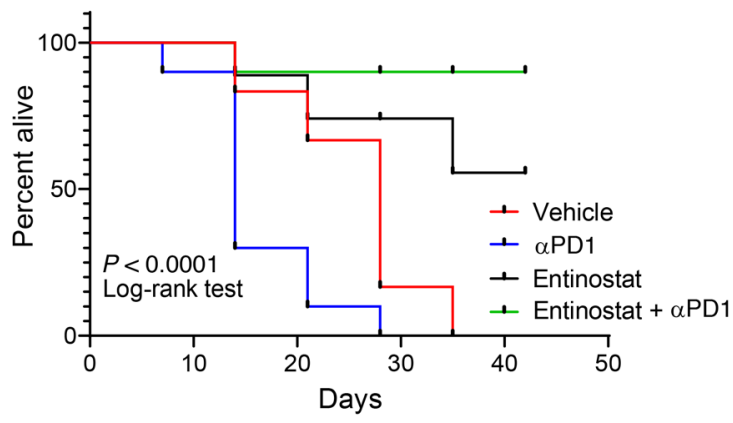

$\mathbf{E}$

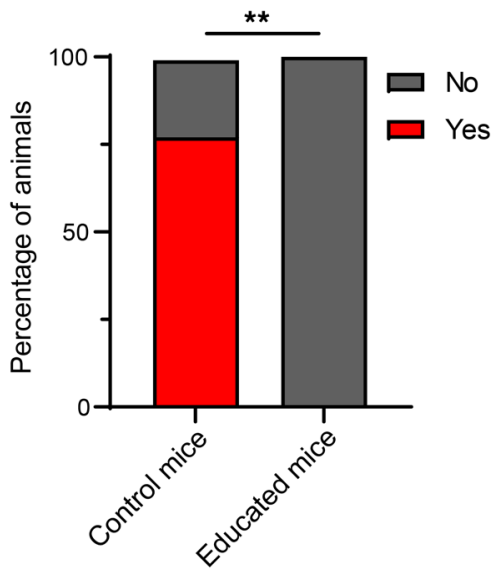

Figure 6. Combination of entinostat and PD-1 inhibition confers effective and durable antitumor immunity. (A) Box plots of PD-L1 (CD274) RNA expression of vehicle- and entinostat-treated BBN963 tumors from C57BL/6 mice. Significance was calculated using Mann-Whitney $U$ test. (B) Average BBN963 tumor volume over time in response to entinostat and anti-PD-1 treatments. Significance was calculated using Wilcoxon's matchedpairs signed rank test. $n=6-10$ mice. (C) Waterfall plot of individual tumor volumes in $\mathbf{B}$ at end point relative to pretreatment baseline. (D) Survival curve of mice receiving entinostat and anti-PD-1 in B. Significance was calculated using log-rank test. End points were tumor burden and ulceration. (E) Bar graph indicating the percentages of control versus educated (entinostat plus anti-PD-1 treated) mice that developed detectable subcutaneous BBN963 tumors a month after rechallenge with BBN963 cells. Significance was calculated using Fisher's exact test. Data are represented as mean \pm SD. ${ }^{* *} P<0.01$.

potent antitumor efficacy of entinostat (particularly when combined with anti-PD-1) in our syngeneic mouse models of bladder cancer and demonstrate for what we believe is the first time that entinostat promotes antitumor responses and immune editing of tumor neoantigens.

The HDAC family consists of 18 potential HDAC enzymes divided into 4 classes (29). While HDACs are best known for their ability to regulate posttranslational acetylation of histone tails affecting gene transcription, research over the past decade has also demonstrated that HDACs regulate the acetylation status of a wide variety of nonhistone proteins in human cells $(29,30)$. Of the HDAC family, class 1 HDACs (HDAC1, -2, -3) appear to be the primary regulators of histone acetylation, while other HDAC classes deacetylate nonhistone substrates (31). Based upon this knowledge, entinostat is predicted to primarily affect transcription.
Prior work in preclinical models has demonstrated that HDAC inhibition affects the tumor immune microenvironment. The majority of these studies attribute this effect to alterations in the balance of intratumoral effector to suppressor immune cells. For example, HDAC inhibition consistently increases the number of $\mathrm{CD}^{+} \mathrm{T}$ cells and also either decreases the number of suppressive immune cells, such as Tregs or MDSCs, or alters their suppressive function $(19,20,25,32,33)$. These effects on antitumor immunity have been studied both in the context of monotherapy and in combination with ICI across diverse models, but not previously, to our knowledge, in bladder cancer. Our data contribute to this literature by demonstrating that entinostat specifically decreases the intratumoral M-MDSC population as well as demonstrating that M-MDSCs, but not G-MDSCs, potently suppress $\mathrm{T}$ cell expansion in vitro. 
The majority of prior studies examining the putative mechanism of action of HDAC inhibitors have demonstrated that HDAC inhibition increases antitumor immunity primarily through non-cell-autonomous means. We therefore chose to focus on the cell-autonomous effects of class 1 HDAC inhibition. Since class 1 HDACs regulate histone acetylation to affect gene transcription, we hypothesized that class 1 HDAC inhibition might affect antitumor immunity through regulation of cancer-associated antigens. Of the known cancer-associated antigens (CTAs, ERVs, and neoantigens) in our studies, only tumor neoantigens were targeted for immune editing in response to entinostat. This work therefore describes a mechanism of action of the class 1 HDAC inhibitor entinostat, triggering changes in the expressed neoantigen landscape.

We are not the first to examine entinostat-mediated, cell-autonomous mechanisms of action. For example, others have shown that HDAC inhibition can promote the induction of a senescent-like phenotype and activation of the senescence-associated secretory phenotype (SASP) through ataxia-telangiectasia mutated (ATM) protein kinase and NF- $\mathrm{KB}$ to promote the recruitment of inflammatory cells (34). In keeping with this result, a screen looking for drugs that induced tumor cell proinflammatory chemokine expression found that HDAC inhibitors induced several chemokines (CCL5, SCSL9, CXCL10; ref. 18).

With the exception of romidepsin, the currently approved HDAC inhibitors (vorinostat, belinostat, and panobinostat) all broadly inhibit the entire HDAC family. In contrast, entinostat is a highly selective class 1 HDAC (HDAC1 and HDAC3) inhibitor. While we ourselves did not perform genetic experiments to determine whether inhibition of HDAC1, HDAC3, or both is necessary for the antitumor effects of entinostat, prior work has shown that upregulation of proinflammatory cytokines in fibroblasts or the antineoplastic effects of vorinostat on cutaneous T cell lymphoma (CTCL) cells were specific to HDAC1 inactivation $(21,34)$. Therefore, while entinostat's selective inhibition of HDAC1 and HDAC3 is certainly an improvement over that of currently approved nonselective inhibitors, one could imagine the development of an HDAC1-selective inhibitor may produce increased efficacy with less toxicity.

We propose that in urothelial bladder cancer, entinostat works through altering the expressed antigen landscape, thereby increasing presentation of immunogenic neoantigens. However, as with most drugs, the majority of patients are unlikely to respond to therapy. Based on our studies, we hypothesize that patients most likely to respond would have a $\mathrm{T}$ cell -inflamed phenotype as well as a high TMB and therefore neoantigens. However, given the poor performance to date of biomarkers predictive of ICI response, it seems realistic to think these features may merely enrich for responders. Moreover, we note that entinostat appears to have effects that are likely independent of the immune microenvironment, given the small effect on tumor-growth inhibition across the multiple BBN models tested in immune-deficient NSG mice. In keeping with this notion, it is notable that we did not see significant antitumor responses to entinostat in our UPPL1541 syngeneic model, which we have previously shown has a low neoantigen burden as well as a non-T cell-inflamed phenotype (Supplemental Figure 8A and ref. 24).

To date, HDAC inhibitors appear to be the most efficacious in the treatment of CTCL, with overall response rates of $30 \%$ and CRs sometimes seen in even heavily pretreated patients. An inter- esting report examined the chromatin accessibility of CTCL using ATAC-Seq from CTCL patients and noted that clinical responses to HDAC inhibitor treatment (vorinostat or romidepsin) were associated with global dynamic increases in chromatin accessibility located in promoters and active enhancers (35). Relevant to our work, HDAC inhibitor treatment did not evoke new accessible elements, but rather greatly accentuated the DNA accessibility of already accessible genomic loci. These findings in part may explain the selectivity of entinostat's effects on immune editing of tumor neoantigens, but not ERVs or CTAs, as neoantigens in general exist in areas of open chromatin in contrast with CTAs and ERVs, which are more generally located in areas of closed chromatin.

Currently, there are several ongoing clinical trials investigating the efficacy of entinostat and ICI combinations in treating various types of solid tumors. In one trial (ClinicalTrials.gov NCT02437136), entinostat and pembrolizumab were given to nonsmall cell lung cancer (NSCLC) and melanoma patients that had progressed on or after PD-1/PD-L1 inhibitors. The combination treatment demonstrated remarkable antitumor activity. In contrast, in trial NCT02915523, in epithelial ovarian cancer patients, the combination entinostat plus avelumab was of no clinical benefit compared with avelumab alone despite the fact that the combination significantly reduced MDSC levels (36). While there are many potential reasons for the discrepant results between these 2 trials, it is notable that the trial with efficacy was in tumors with a high TMB (NSCLC, melanoma), while the negative trial was in ovarian cancer, which has a relatively low TMB (7). We used the IMvigor210 cohort 1 (chemo naive) and cohort 2 (prior chemo) to assess any potential interaction between chemotherapy exposure and $\mathrm{TMB} /$ neoantigen burden. Consistent with previously published work, we did not see a difference in either cohort (Supplemental Figure 8B) These findings are in keeping with the idea that entinostat's antitumor effects rely on tumor neoantigen editing.

In summary, our study demonstrates a cell-autonomous mechanism of action for the selective HDAC1 and -3 inhibitor entinostat, altering the expressed antigen landscape. We show that entinostat, when combined with PD-1 axis blockade, induces complete remission of tumors in mice, expansion of neoantigen-specific $\mathrm{T}$ cells, and induction of long-term immunologic memory. Published work examining the effect of HDAC inhibition on chromatin accessibility in CTCL may explain the relative specificity of the immune editing to neoantigens and not CTAs or ERVs seen in our models. The preclinical efficacy of entinostat and antiPD-1 combination seen in our hands has provided the rationale for the initiation of a window-of-opportunity clinical trial evaluating the combination of entinostat and pembrolizumab in cisplatin-ineligible, muscle-invasive bladder cancer patients (NCT03978624) undergoing cystectomy. This effort will be important to validate whether our paradigm is paralleled in humans.

\section{Methods}

Cell culture. UPPL1541, BBN963, and BBN966 mouse bladder cancer cell lines were generated as previously described (24). All cell lines were cultured in DMEM (MilliporeSigma) supplemented with L-glutamine, $10 \%$ fetal bovine serum, and $1 \times$ penicillin/streptomycin (Gibco, Thermo Fisher Scientific). All cell lines were maintained at $37^{\circ} \mathrm{C}$ in $5 \% \mathrm{CO}_{2}$. Mycoplasma testing was performed routinely while cells were in culture. 
Cell-proliferation assay. To determine $\mathrm{IC}_{50}$ values, cell lines were plated in triplicate in 96-well plates at 500 cells/well and treated with increasing concentrations of entinostat or vehicle. Cell numbers were determined on day 3 using the CellTiter-Glo Luminescent Cell Viability assay per the manufacturer's instructions (Promega). $\mathrm{IC}_{50}$ values were derived from the 9 dose-response curves.

Western blot. Cells were lysed in 1× RIPA buffer (Abcam) supplemented with protease inhibitor cocktail (Roche) and phosphatase inhibitor cocktail (Calbiochem). Cell extracts (20-30 $\mu \mathrm{g}$ ) were resolved by SDS-PAGE, transferred to a nitrocellulose membrane, and then probed with the indicated antibodies (Supplemental Table 1). Proteins were visualized with the chemiluminescence system from Amersham using HRP-conjugated anti-rabbit (catalog 31462) or antimouse (catalog 31432) secondary antibodies (Thermo-Scientific).

Gene-expression profiling. Whole transcriptome profiles were generated from mouse tumors. RNA was extracted using the QIAGEN RNeasy Plus Mini Kit. RNA-Seq libraries were prepared using the TruSeq Stranded mRNA Library Preparation Kit (Illumina) according to the manufacturer's protocol. Sequencing was run on Nextseq 500 (Illumina). RNA reads were aligned to the human reference genome GRCh38.p5 (Gencode) using STAR (version 2.5.3a). The transcript levels were then quantified using SALMON (version 0.9.1). RNA-Seq data were deposited in the NCBI's Gene Expression Omnibus database (GEO GSE153650).

Differential gene expression. Count data were extracted from SALMON output using Tximport (Bioconductor) and normalized and compared using DESeq2 (Bioconductor).

WES. DNA was extracted by using the DNeasy Blood and Tissue Kit (QIAGEN). Whole-exome library preparation was performed using the Agilent SureSelect XT Mouse All Exon Kit. Libraries were sequenced via $2 \times 100$ runs on an Illumina HiSeq 2500 at the UNC High Throughput Sequencing Facility. DNA reads of tumor and matched normal tissue were aligned to mouse genome $\mathrm{mm} 9$ by bwa (version 0.7.4). Duplicates were removed, and bam files were sorted and indexed by Picard (version 1.96). Somatic mutations of tumor samples were identified by Strelka and annotated using snpEff (version 3.3). WES data were deposited in the NCBI's GEO database (GEO GSE153650).

Neoantigen analysis. Bioinformatics prediction of neoantigens was performed as previously described (37) using RNA-Seq fastq files from above and mutation files from above and a previous study (24). netMHCpan-4.0 (38) was then used to predict HLA peptide-binding affinity for somatic mutation-derived variant peptides with a length between 8 and 11 amino acids. HLA (H-2-Db or H-2-Kb) peptides with binding affinity higher than $500 \mathrm{nM}$ were considered as nonbinders. Predicted neoantigens were filtered on expression in all replicates with greater than 5 read support

TCR-Seq analysis. RNA was extracted using the QIAGEN RNeasy Plus Mini Kit. TCR libraries were prepared using the SMARTer Mouse TCR a/b Profiling Kit (TaKaRa) according to the manufacturer's instructions. Sequencing was performed on a MiSeq (Illumina). TCR amplicon data were analyzed via MiXCR to generate TCR clonotype expression matrices for each sample. Data were converted into standard in-lab format, and downstream analysis was performed with custom scripts as well as the tcR package (39).

Flow cytometry. Tumor sample processing, preparation, and analysis were performed as previously described (24). In brief, tumor tissues were collected and immediately homogenized in cold media using the GentleMACS Dissociator. After passing through a $70 \mu \mathrm{m}$ cell strainer, the samples were treated with ACK lysis buffer to remove red blood cells. The samples were then washed, counted, and stained with FVS700 viability stain and antibody master mix (antibodies listed in Supplemental Table 2). Cells were incubated on ice in the dark for 45 minutes and washed twice with staining buffer. Cells were fixed in $2 \%$ paraformaldehyde overnight. Subsequently, a minimum of 100,000 events were collected for each sample on a BD LSRFortessa Flow Cytometer. FlowJo software was used for analyses. Single color and fluorescence minus one controls were used to guide gating strategies. qRT-PCR. RNA was extracted using the QIAGEN RNeasy Plus Mini Kit according to the manufacturer's instructions. The cDNA library was synthesized with random primers, using an ImProm-II reverse transcription system (Promega). qPCR was performed using iTaq Universal SYBR Green Supermix (Bio-Rad) on a ViiA 7 Real-Time PCR System according to the manufacturer's instructions. The list of primers used can be found in Supplemental Table 3. The results were analyzed using the $\Delta \Delta \mathrm{Ct}$ relative quantification method.

CRISPR/Cas9. The RNA guides were designed using Broad Institute sgRNA Designer. The guides were cloned into lentiCRISPRv2 blast vector. The lentiCRISPRv2 blast was a gift from Brett Stringer (Addgene, plasmid 98293).

Mouse allograft model and in vivo treatment. BBN963, BBN966, and BBN963_sgB2m, BBN963_EV cell lines were injected subcutaneously into 6- to 10-week-old female C57BL/6 mice (Charles River Laboratories) at $3.0 \times 10^{6}$ cells. Once tumors reached $200 \mathrm{~mm}^{3}$ in tumor volume, mice were randomized and treated with chow containing entinostat $(12 \mathrm{mg} / \mathrm{kg})$ or given an intraperitoneal injection with anti-PD-1 antibody (Bio X Cell, clone RMP1-14) or a combination of entinostat and anti-PD-1 or a drug-free control chow. Tumor volume and body weight were measured weekly for the duration of the treatment. Mice were sacrificed if body weight decreased more than $10 \%$ of the initial weight, tumor volume reached $1000 \mathrm{~mm}^{3}$, or there was skin ulcer formation.

To rechallenge the mice with tumor cell injection, mice that had CR continued to be on treatments for a month after reaching CR. After the last treatment, the animals continued to be monitored for at least another month before being rechallenged. To challenge the mice, BBN963 cells were injected subcutaneously in these animals at $3.0 \times 10^{6}$ cells. Animals were monitored weekly for a month to determine the take rate, which was defined by the presence of tumors.

$T$ cell suppression assay. $\mathrm{T}$ cells from naive (WT) mice were isolated from spleens of animals, labeled with $5 \mu \mathrm{M}$ CFSE, and used as responder cells in all experiments. Ninety-six-well flat-bottom culture plates were precoated with $100 \mu \mathrm{l}$ of a mixture of $3 \mu \mathrm{g} / \mathrm{ml}$ anti-CD3 (InVivoMAb, clone 145-2C11) and $5 \mu \mathrm{g} / \mathrm{ml}$ anti-CD28 (InVivoMAb, clone 37.51) overnight at $4^{\circ} \mathrm{C}$ prior to the experiment. The plate was flicked to remove solution prior to adding cells in media. A separate plate was used for no-stimulation controls. CFSE-labeled T cells were cultured separately with either $\mathrm{Ly}_{6} \mathrm{C}^{+}$(termed $\mathrm{Gr}^{+}$in these experiments) or Ly $6 \mathrm{G}^{+}$cells for 72 hours.

25,000 CFSE-labeled T cells in $100 \mu \mathrm{l}$ were added to each well of the anti-CD3/anti-CD28-coated plates and no-stimulation plate. The suppressive population of cells in $100 \mu \mathrm{l}$ was then added to each well containing T cells for a total of $200 \mu$ final volume during the 72-hour culture period. Proliferation of T cells was read out as CFSE dilution on cytometer after culture period. All conditions were cultured in triplicate. 
T cell killing assay. BBN963 cells were treated with DMSO or $1 \mu \mathrm{M}$ entinostat for 72 hours in vitro. Cells were detached by using Accutase and washed with PBS. To each well of a 96-well plate, 4000 cells were seeded. The CD8 ${ }^{+} \mathrm{T}$ cells were collected from the spleen of BBN963 tumor-bearing mice that either had or had not been treated with entinostat for 4 weeks and cultured for 48 hours in the presence of soluble anti-CD28 (InVivoMAb, clone 37.51) and plate-bound anti-CD3 (InVivoMAb, clone 145-2C11). T cells were collected and resuspended in fresh media. To each corresponding well of the 96-well plate in which BBN963 cells were plated, 40,000 T cells were subsequently seeded. The cells were cocultured for 72 hours. T cells were washed out. The viability of BBN963 cells was determined by CellTiter-Glo.

Tetramer labeling. Tetramers were generated using the MBL Quickswitch Quant H-2 Kb Tetramer Kit-PE (catalog TB-7400-K1) according to the manufacturer's protocol. Peptides were synthesized and purchased from New England Peptide. The list of neoantigens can be found in Supplemental Table 4. C57BL/6 mice bearing BBN963 tumors were treated with either vehicle or entinostat chow $(12 \mathrm{mg} /$ $\mathrm{kg}$ ) for 3 weeks. Splenocytes were isolated from these mice and treated with $50 \mathrm{nM}$ dasatinib for 30 minutes at $37^{\circ} \mathrm{C}$. Approximately $5 \times$ $10^{6}$ cells were stained with $5 \mu \mathrm{g} / \mathrm{mL}$ tetramer on ice for 30 minutes. Cells were then washed and stained with the following markers: CD3 (1 $\mu$ g; FITC; BioLegend, clone 17A2) and CD8 (0.5 $\mu \mathrm{g}$; AF647; Bio-Rad, clone KT15). Finally, cells were washed and stained for viability using the Zombie UV Fixable Viability Kit (BioLegend 423107) according to the manufacturer's instructions. Acquisition was performed using a BD LSRFortessa flow cytometer. FlowJo software was used for analyses of the data. Single color and fluorescence-minus-one controls were used to guide gating strategies. Tetramer-positive $\mathrm{CD} 8^{+} \mathrm{T}$ cells were defined within singlet (FSC-H vs FSC-W), live, $\mathrm{CD}^{+}$, and $\mathrm{CD}^{+}$gates.

Statistics. Statistical analyses were performed using GraphPad Prism, version 8.0. Two-tailed $t$ tests or Mann-Whitney $U$ tests were used to compare 2 groups, while 3 or more groups were compared using 2-way ANOVA followed by either Tukey's or Dunnett's multiple-comparison test. A $P$ value of less than 0.05 was considered significant.
Study approval. All animal studies were reviewed and approved by The UNC IACUC. No human studies requiring IRB approval were performed.

\section{Author contributions}

AST, MZ, BGV, TLR, MIM, WB, and WYK were responsible for overall study concept, design of experiments, and final data interpretation. AST, MZ, BK, TU, KGS, CCS, and LMB acquired and analyzed data. STB, SEW, XH, CMP, and UM provided technical support. MZ provided bioinformatics support. All authors provided helpful discussions and feedback toward drafting the manuscript.

\section{Acknowledgments}

We acknowledge the members of the Kim Lab for useful discussions and funding from the Thomas M. Mohr Fund for Bladder Cancer Research and the Waterproof Foundation that helped support this research. We thank the UNC Mouse Phase 1 Unit (MP1U), UNC Translational Pathology Laboratory, and Center for Gastrointestinal Biology and Disease (CGIBD) Facility for their technical assistance. This work was supported by the University Cancer Research Fund (UCRF) (to WYK and BGV), American Cancer Society grant RSG-14-219-01-TBG (to WYK), National Cancer Institute R01-CA241810 (to WYK and BGV), the Uhera Foundation (to TU), and NCI F31 CA247250 (AST). TLR is supported by National Cancer Institute K08CA248967 as well as the Doris Duke Charitable Foundation (grant 2015213). BGV was supported by the National Cancer Institute at the NIH K12 Career Development Award in Clinical Oncology (grant 5K12CA120780). The graphical abstract was made with BioRender.

Address correspondence to: William Y. Kim or Benjamin G. Vincent, Lineberger Comprehensive Cancer Center, University of North Carolina, CB\# 7295, Chapel Hill, North Carolina 27599-7295, USA. Phone: 919.966.4765; Email: wykim@med.unc.edu (WYK); Phone: 919.966.8412; Email: Benjamin_vincent@med.unc.edu (BGV).
1. Siegel RL, et al. Cancer Statistics, 2021. CA Cancer JClin. 2021;71(1):7-33.

2. Bellmunt J, et al. Pembrolizumab as second-line therapy for advanced urothelial carcinoma. N Eng J Med. 2017;376(11):1015-1026.

3. Plimack ER, et al. Safety and activity of pembrolizumab in patients with locally advanced or metastatic urothelial cancer (KEYNOTE-012): a non-randomised, open-label, phase $1 \mathrm{~b}$ study. Lancet Oncol. 2017;18(2):212-220.

4. Sharma $P$, et al. Nivolumab monotherapy in recurrent metastatic urothelial carcinoma (CheckMate 032): a multicentre, open-label, two-stage, multi-arm, phase $1 / 2$ trial. Lancet Oncol. 2016;17(11):1590-1598.

5. Rosenberg JE, et al. Atezolizumab in patients with locally advanced and metastatic urothelial carcinoma who have progressed following treatment with platinum-based chemotherapy: a single-arm, multicentre, phase 2 trial. Lancet . 2016;387(10031):1909-1920.

6. Balar AV, et al. Atezolizumab as first-line treatment in cisplatin-ineligible patients with locally advanced and metastatic urothelial carcinoma: a single-arm, multicentre, phase 2 trial. Lancet 2017;389(10064):67-76.

7. Alexandrov LB, et al. Signatures of mutational processes in human cancer. Nature. 2013;500(7463):415-421.

8. Schumacher TN, Schreiber RD. Neoantigens in cancer immunotherapy. Science. 2015;348(6230):69-74.

9. Rizvi NA, et al. Cancer immunology. Mutational landscape determines sensitivity to PD-1 blockade in non-small cell lung cancer. Science. 2015;348(6230):124-128.

10. Yarchoan M, et al. Tumor mutational burden and response rate to $\mathrm{PD}-1$ inhibition. $N$ Engl J Med. 2017;377(25):2500-2501.

11. Litchfield $\mathrm{K}$, et al. Meta-analysis of tumor- and $\mathrm{T}$ cell-intrinsic mechanisms of sensitization to checkpoint inhibition. Cell. 2021;184(3):596-614.

12. Seto E, Yoshida M. Erasers of histone acetylation: the histone deacetylase enzymes. Cold Spring Harb Perspect Biol. 2014;6(4):a018713.

13. Robertson AG, et al. Comprehensive molecular characterization of muscle-invasive bladder cancer. Cell. 2017;171(3):540-556.
14. Mitsiades CS, et al. Transcriptional signature of histone deacetylase inhibition in multiple myeloma: biological and clinical implications. Proc Natl Acad Sci U S A. 2004;101(2):540-545.

15. Gui CY, et al. Histone deacetylase (HDAC) inhibitor activation of p21WAF1 involves changes in promoter-associated proteins, including HDAC1. Proc Natl Acad Sci U S A. 2004;101(5):1241-1246.

16. Lee JH, et al. Histone deacetylase inhibitor enhances 5-fluorouracil cytotoxicity by down-regulating thymidylate synthase in human cancer cells. Mol Cancer Ther. 2006;5(12):3085-3095.

17. $\mathrm{Xu} \mathrm{W}$, et al. Intrinsic apoptotic and thioredoxin pathways in human prostate cancer cell response to histone deacetylase inhibitor. Proc Natl Acad SciU S A. 2006;103(42):15540-15545.

18. Zheng $\mathrm{H}$, et al. HDAC inhibitors enhance T-cell chemokine expression and augment response to PD-1 immunotherapy in lung adenocarcinoma. Clin Cancer Res. 2016;22(16):4119-4132.

19. Kim K, et al. Eradication of metastatic mouse cancers resistant to immune checkpoint blockade by suppression of myeloid-derived cells. Proc Natl Acad Sci U S A. 2014;111(32):11774-11779. 
20. Orillion A, et al. Entinostat neutralizes myeloid-derived suppressor cells and enhances the antitumor effect of PD-1 inhibition in murine models of lung and renal cell carcinoma. Clin Cancer Res. 2017;23(17):5187-5201.

21. Gameiro SR, et al. Inhibitors of histone deacetylase 1 reverse the immune evasion phenotype to enhance T-cell mediated lysis of prostate and breast carcinoma cells. Oncotarget. 2016;7(7):7390-7402.

22. Juergens RA, et al. Combination epigenetic therapy has efficacy in patients with refractory advanced non-small cell lung cancer. Cancer Discov. 2011;1(7):598-607.

23. Zhu S, et al. The narrow-spectrum HDAC inhibitor entinostat enhances NKG2D expression without NK cell toxicity, leading to enhanced recognition of cancer cells. Pharm Res. 2015;32(3):779-792.

24. Saito R, et al. Molecular subtype-specific immunocompetent models of high-grade urothelial carcinoma reveal differential neoantigen expression and response to immunotherapy. Cancer Res. 2018;78(14):3954-3968.

25. Shen L, et al. Class I histone deacetylase inhibitor entinostat suppresses regulatory $\mathrm{T}$ cells and enhances immunotherapies in renal and prostate cancer models. PLoS One. 2012;7(1):e30815.

26. Chen DS, Mellman I. Oncology meets immunology: the cancer-immunity cycle. Immunity. 2013;39(1):1-10.

27. McGranahan N, Swanton C. Cancer evolution constrained by the immune microenvironment. Cell. 2017;170(5):825-827.

28. Keenan TE, et al. Genomic correlates of response to immune checkpoint blockade. Nat Med. 2019;25(3):389-402.

29. Li Y, Seto E. HDACs and HDAC inhibitors in cancer development and therapy. Cold Spring Harb Perspect Med. 2016;6(10):a026831.

30. Choudhary C, et al. Lysine acetylation targets protein complexes and co-regulates major cellular functions. Science. 2009;325(5942):834-840.

31. West AC, Johnstone RW. New and emerging HDAC inhibitors forcancer treatment. J Clin Invest. 2014;124(1):30-39.

32. Christmas BJ, et al. Entinostat converts immune-resistant breast and pancreatic cancers into checkpoint-responsive tumors by reprogramming tumor-infiltrating MDSCs. Cancer Immunol Res. 2018;6(12):1561-1577.

33. Kato $S$, et al. Hyper-progressors after immuno- therapy: analysis of genomic alterations associated with accelerated growth rate. Clin Cancer Res. 2017;23(15):4242-4250.

34. Pazolli E, et al. Chromatin remodeling underlies the senescence-associated secretory phenotype of tumor stromal fibroblasts that supports cancer progression. Cancer Res. 2012;72(9):2251-2261.

35. Qu K, et al. Chromatin accessibility landscape of cutaneous $\mathrm{T}$ cell lymphoma and dynamic response to HDAC inhibitors. Cancer Cell. 2017;32(1):27-41.

36. Cadoo KA, et al. A phase II randomized study of avelumab plus entinostat versus avelumab plus placebo in patients (pts) with advanced epithelial ovarian cancer (EOC). JClin Oncol. 2019;37(15_Suppl):5511-5511.

37. Kardos J, et al. Claudin-low bladder tumors are immune infiltrated and actively immune suppressed. JCI Insight. 2016;1(3):e85902.

38. Jurtz V, et al. NetMHCpan-4.0: improved peptide-MHC class I interaction predictions integrating eluted ligand and peptide binding affinity data. J Immunol. 2017;199(9):3360.

39. Nazarov VI, et al. tcR: an R package for T cell receptor repertoire advanced data analysis. $B M C$ Bioinformatics. 2015;16(1):175. 